Article

\title{
Secreted Frizzled-Related Protein Promotes Bone Regeneration by Human Bone Marrow-Derived Mesenchymal Stem Cells
}

\author{
Wataru Katagiri *, Masashi Osugi, Takamasa Kawai and Hideharu Hibi \\ Department of Oral and Maxillofacial Surgery, Nagoya University Graduate School of Medicine, \\ 65 Tsuruma-cho, Showa-ku, Nagoya 466-8550, Japan; E-Mails: masashi@med.nagoya-u.ac.jp (M.O.); \\ takamacha1@hotmail.com (T.K.); hibihi@med.nagoya-u.ac.jp (H.H.) \\ * Author to whom correspondence should be addressed; E-Mail: w-kat@med.nagoya-u.ac.jp; \\ Tel.: +81-527-442-348; Fax: +81-527-442-352.
}

Academic Editor: Wilhelm Bloch

Received: 4 August 2015 / Accepted: 23 September 2015 / Published: 25 September 2015

\begin{abstract}
Secreted frizzled-related protein (sFRP)-3 is a negative regulator of Wnt signaling in human mesenchymal stem cells (hMSCs). The present study investigated the effects sFRP-3 on osteogenic differentiation by assessing osteogenic gene expression in hMSCs in vitro and by examining bone regeneration in a rat bone defect model. sFRP-3 treatment induced osteogenic differentiation in hMSCs as determined by alkaline phosphatase, collagen type I, osteocalcin, and Runt-related transcription factor 2 gene expression. hMSCs with or without sFRP-3 were implanted into a rat calvarial bone defect; a radiographic analysis by micro-computed tomography and histological analysis 4 and 8 weeks after implantation showed greater bone regeneration in the $\operatorname{sFRP}(+)$ than in the sFRP(-) group. These results suggest that modulation of Wnt signaling contributes to osteogenic differentiation in hMSCs. Specifically, sFRP-3 induces osteoblastic differentiation of cultured MSCs and bone regeneration in a calvarial bone defect, suggesting that it can be a useful agent for the treatment of bone defects.
\end{abstract}

Keywords: human mesenchymal stem cells (hMSCs); secreted frizzled-related protein-3 (sFRP-3); Wnt signaling; osteogenic differentiation; bone regeneration 


\section{Introduction}

Until recently, autogenous bone grafts were considered the gold standard for the treatment of bone defects resulting from surgery, trauma, and inflammatory disease. This well-studied procedure has good prognosis but requires an extra surgery at the donor site, which is an additional burden on the patient. Although several bone substitutes are commercially available, these materials tend to be easily infected and induce antigenic responses. An alternative treatment strategy for bone defects is growth factor therapy; factors such as bone morphogenetic protein 2 have demonstrated excellent osteogenic potential [1,2] and are already available on the market. However, high physiological doses are required to produce an effect [3] and a severe inflammatory response may be induced [4,5], while the amount of bone that can be regenerated is limited. Therefore, there is a need to establish novel bone regeneration techniques that circumvent these issues.

Tissue engineering involves the generation of new tissue from isolated cells with scaffolds and growth factors [6]. While pluripotent stem cells such as embryonic stem cells and induced pluripotent stem cells have a greater potential for regenerative medicine, adult stem cells are currently more widely used for therapeutic applications owing to ethical and safety considerations. Among the various types of adult stem cell, bone marrow-derived mesenchymal stem cells (MSCs) have been extensively studied and used in various therapeutic applications. We previously used a mixture of human (h)MSCs and platelet-rich plasma (PRP) (hMSCs/PRP) — bone graft materials with predictable grafting success - for craniofacial reconstruction and dental implants [7], and bone regeneration using hMSCs/PRP has been successful in some clinical settings [8]. hMSCs can be obtained by a minimally invasive technique and their use can obviate the need for donor site surgery in autogenous bone graft procedures. However, there are problems associated with hMSC-based tissue regeneration, such as the variability in terms of cell proliferation and differentiation time among patients. A more effective osteogenic induction method that reduces culture time while enhancing bone quantity and quality is desired.

Wnts constitute a large family of secreted signaling molecules that play a number of important roles during animal development [9]. The canonical Wnt signaling pathway regulates cytoplasmic accumulation of $\beta$-catenin, leading to the downstream transcription of Wnt target genes [10]. Canonical Wnt signaling is known to be involved in the self-renewal of hematopoietic stem cells [11] and has been implicated in bone formation; for instance, osteoporosis pseudoglioma syndrome is associated with an inactivating mutation in the Wnt co-receptor low-density lipoprotein receptor-related protein $5[12,13]$, providing evidence that canonical Wnt signaling is a key regulator of osteogenesis.

Wnt signals are also important for hMSC proliferation and differentiation. Wnt 3a, a canonical Wnt member, induces $\beta$-catenin nuclear localization and MSC proliferation while inhibiting osteogenic differentiation. Low concentrations of the Wnt mimic lithium, which inhibits phosphorylation of $\beta$-catenin by GSK-3 $\beta$, also stimulates hMSC proliferation [14-16]. On the other hand, the Wnt inhibitor secreted Frizzled-related protein (sFRP)-3 is upregulated in MSC osteogenesis [14]. sFRP-3 was shown to induce osteoblastic differentiation of hMSCs by binding Wnt5A and thereby antagonizing non-canonical Wnt signaling [17]. The modulation of Wnt signaling by sFRP-3 has therapeutic implications for the treatment of bone defects, since it may accelerate regeneration and thus shorten the treatment period and reduce the cost of cell culture. However, little is known about the 
in vivo effects of sFRP-3-treated hMSCs on bone regeneration. The present study investigated the role of sFRP-3 in the osteogenic potential of hMSCs in vitro and on bone regeneration in vivo in a rat calvarial bone defect model.

\section{Results and Discussion}

\section{1. sFRP-3 Induces the Expression of Osteogenic Markers in hMSCs}

The expression of ALP, collagen type I (Coll), osteocalcin (OC), and Runt-related transcription factor (Runx)2 was assessed relative to glyceraldehyde 3-phosphate dehydrogenase (GAPDH) by quantitative real-time (qRT-)PCR on day 14 in hMSCs cultured with or without sFRP-3. The mRNA levels of each of these genes associated with osteogenic differentiation were upregulated in hMSCs treated with sFRP-3 as compared to untreated cells (Figure 1).
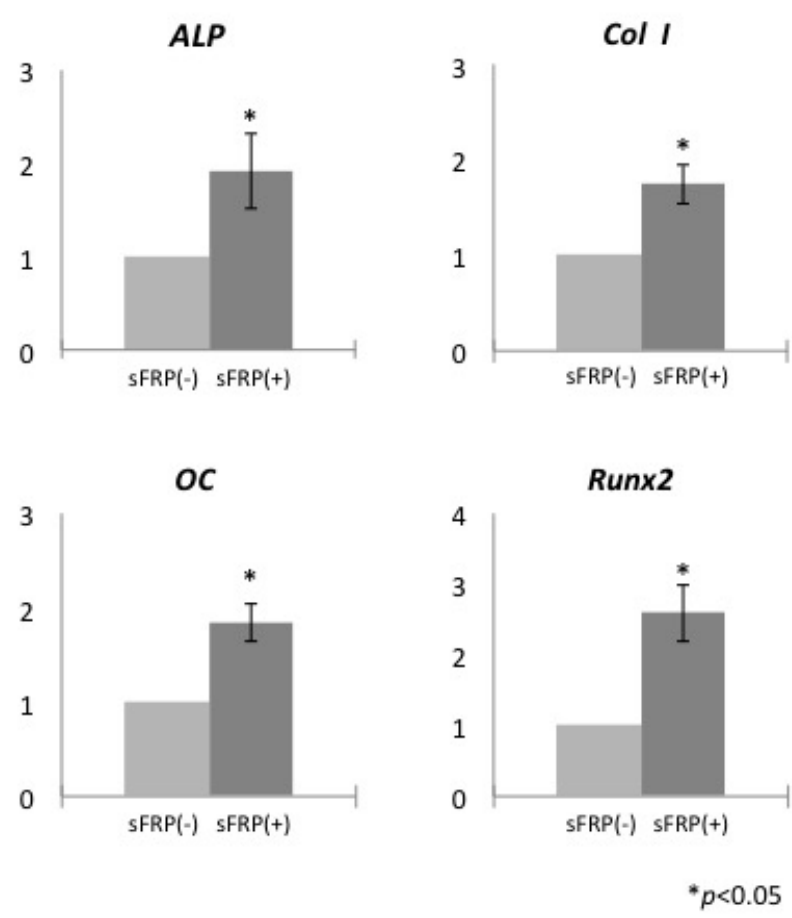

Figure 1. Relative mRNA expression of osteogenesis-related genes in human mesenchymal stem cells (hMSCs) cultured with or without secreted frizzled-related protein (sFRP-3). $* p<0.05$.

sFRP-3 belongs to a family of soluble proteins that are structurally related to Frizzled Wnt receptors; indeed, they are considered as decoy receptors that bind to Wnt proteins to prevent signal activation [18]. sFRP-1 inhibits Wnt- $\beta$-catenin signaling in osteoblasts, resulting in the loss of bone mass in adult mice, and sFRP-1-null mice exhibit increased trabecular bone mineral density, volume, and mineral apposition rate as compared to controls [19]. Human multiple myeloma cells constitutively produce sFRP-2 and suppress bone formation at least in part via inhibition of Wnt signaling [20]. sFRP-3 and -4 exhibit opposite effects on osteoblastic differentiation of hMSCs: the former promotes whereas the latter suppresses this process [21]. Thus, sFRP is critical for bone formation and acts primarily by antagonizing Wnt signaling. 
The effects of Wnt signaling differ according to cell background and origin. For example, ALP was activated in $\mathrm{C} 3 \mathrm{H} / 10 \mathrm{~T} 1 / 2$ cells from mouse embryos [22] but inactivated in hMSCs [14,15] by Wnt signaling. Thus, the effects of Wnt signaling on cellular proliferation and differentiation are cell type-specific although the mechanistic basis for these differences is poorly understood. It was previously demonstrated that $A L P$ and bone sialoprotein expression was downregulated in hMSCs induced with Wnt3a but upregulated in the presence of both Wnt3a and sFRP-3 [14]. In addition, transfection of a short interfering RNA against sFRP-3 suppressed ALP activity [21]. These findings along with the present results indicate that Wnt signaling negatively regulates while sFRP-3 activates osteogenic differentiation in hMSCs. In this study, sFRP-3 was used for accelerating osteogenic differentiation of hMSCs by inhibiting Wnt- $\beta$-catenin pathway. We found $10 \mathrm{ng} / \mathrm{mL}$ of sFRP-3 had an inhibitory effect on the cellular proliferation by bromodeoxyuridine (BrdU) staining assay (Figure S1). Furthermore, this inhibitory effect on cellular proliferation was neutralized by the addition of Wnt mimic lithium chloride ( $\mathrm{LiCl}$ ) (Figure S2). Other study showed that Wnt mimic LiCl (4 mM) inhibited osteogenic differentiation of hMSCs [15] and our preliminary study also indicated that the addition of $4 \mathrm{mM}$ of $\mathrm{LiCl}$ accelerated the growth rate of hMSCs (data not shown). Immunocytochemistry also revealed the effects of sFRP-3 and $\mathrm{LiCl}$ on Wnt- $\beta$-catenin pathway in hMSCs. The addition of $\mathrm{LiCl}$ enhanced the localization of $\beta$-catenin in the nucleus, whereas the addition of sFRP-3 inhibited the localization of $\beta$-catenin in the nucleus (Figure S3). Thus, sFRP-3 regulated Wnt- $\beta$-catenin pathway negatively and $\mathrm{LiCl}$ regulated positively in hMSCs. We also found that calcification and ALP activity are increased in hMSCs upon sFRP-3 treatment (Figure S4), as the previous study reported [21].

Osteogenesis-related genes are targets of Wnt signaling; this has been demonstrated by the identification of a binding site for $\mathrm{T}$ cell factor - a transcriptional co-activator of canonical Wnt signaling - in the promoter of the Runx2 gene that functions in bone formation [23]. We found that the expression of ALP, ColI, OC, and Runx2 was upregulated in sFRP-3-treated cells on day 14, providing evidence that inhibition of Wnt signaling by sFRP-3 promotes osteogenic differentiation of hMSCs.

\section{2. sFRP-3 Enhances Bone Regeneration in Vivo}

To determine whether sFRP-3 can induce osteogenic differentiation of hMSCs in vivo, we transplanted hMSCs/Terudermis or hMSCs/sFRP-3/Terudermis $(\operatorname{sFRP}(-)$ and $(+)$, respectively) into rat calvarial bone defects and measured the area of newly regenerated bone as a percentage of the total graft area at 2, 4 and 8 weeks post-transplantation by micro-computed tomography (micro-CT) (Figure 2A). The area of newly regenerated bone was greater in the $\operatorname{sFRP}(+)$ than in the $\mathrm{sFRP}(-)$ group at 4 weeks $(62.9 \% \pm 6.9 \%$ vs. $40.5 \% \pm 9.3 \%)$ and at 8 weeks $(71.9 \% \pm 8.9 \%$ vs. $44.1 \% \pm 11.5 \%)$, although there was no significant difference at 2 weeks $(29.7 \% \pm 8.4 \%$ vs. $31.5 \% \pm 9.4 \%)$ (Figure 2B).

A histological analysis revealed that bone was regenerated to a greater degree in the $\mathrm{sFRP}(+)$ as compared to the sFRP(-) group at 4 and 8 weeks. At 4 weeks, the bone defect was partially covered with newly regenerated bone in the $\operatorname{sFRP}(+)$ group and with connective tissue in the $\operatorname{sFRP}(-)$ group. At 8 weeks, there was some newly regenerated bone detected within the defect in the sFRP(-) group, but the defect was filled with mature bone tissue in the $\operatorname{sFRP}(+)$ group. At 2 weeks, post surgical swelling was seen in each specimen with little bone tissue regeneration (Figure 3). These findings from micro-CT and histological analysis indicated that hMSCs with or without sFRP-3 treatment themselves 
survived keeping their cellular osteogenic characteristics and mineralized after removal of inflammatory tissue observed at 2 weeks.
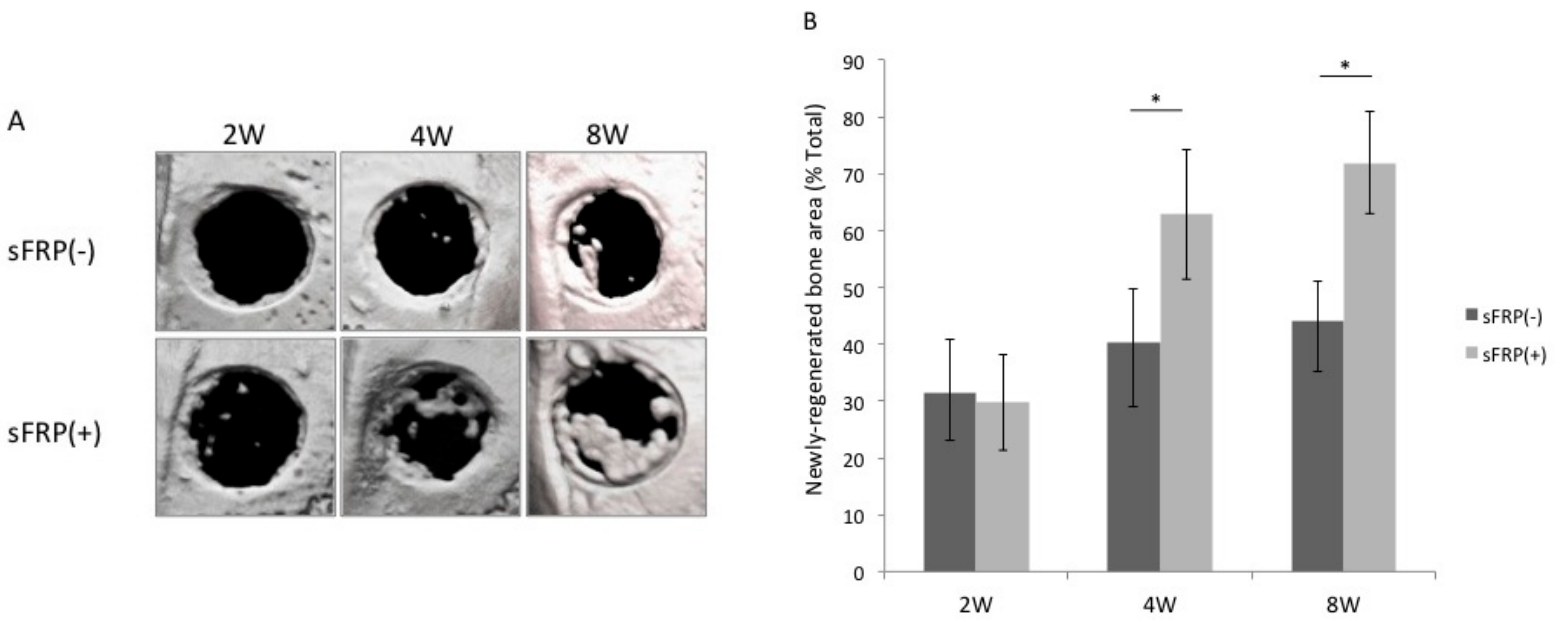

Figure 2. Micro-computed tomography (Micro-CT) analysis of bone regeneration in a rat calvarial bone defect model. (A) Micro-CT images of the bone 2, 4 and 8 weeks after transplantation of hMSCs/Terudermis or hMSCs/sFRP-3/Terudermis (sFRP(-) and $(+)$, respectively); (B) Area of newly regenerated bone $\left(\mathrm{mm}^{2}\right)$ in each defect 2, 4 and 8 weeks after transplantation expressed as a percentage of the entire defect area. $* p<0.05$.

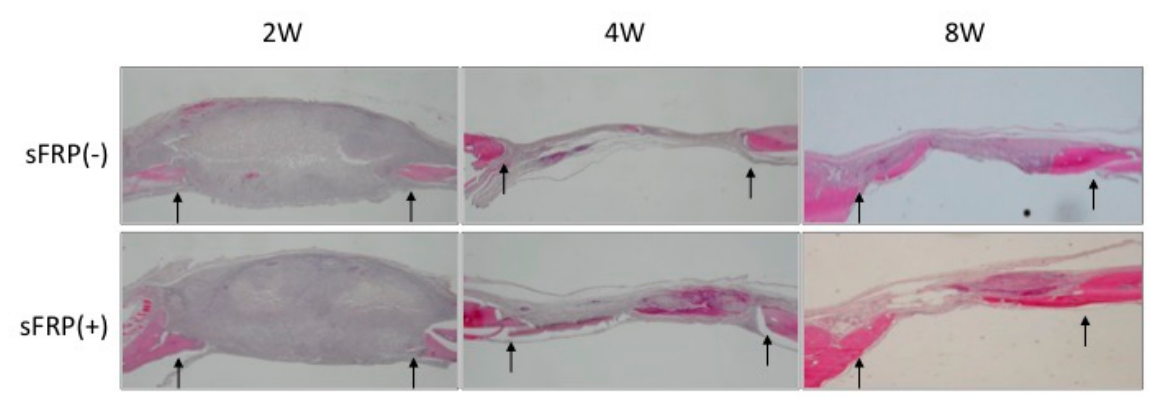

Figure 3. Histological evaluation of newly regenerated calvarial bone in rats. hMSCs with or without sFRP treatment were transplanted into rats and tissue sections were examined 2, 4 and 8 weeks later by staining with hematoxylin and eosin $(12.5 \times)$. Arrows indicate the edges of the bone defect.

The stimulation of bone regeneration by sFRP-3 suggests that Wnt signaling normally inhibits osteogenic differentiation in vitro and bone regeneration in vivo. The inclusion of SFRP-3 in osteogenic cultures of hMSCs can therefore shorten culture time and promote early bone regeneration in clinical applications.

\section{Experimental Section}

\subsection{Cell Culture}

Commercially available human primary marrow-derived MSCs (Lonza, Walkersville, MD, USA) were cultured at $37{ }^{\circ} \mathrm{C}$ in a $5 \% \mathrm{CO}_{2}$ atmosphere in MSCGM consisting of MSC basal medium 
and SingleQuots mesenchymal cell growth supplement, L-glutamine, and penicillin/streptomycin (Lonza). The hMSC osteogenic differentiation Bullet kit (Lonza) was used for osteogenic induction, which contained dexamethasone, ascorbate, mesenchymal cell growth supplement, L-glutamine, penicillin/streptomycin, and $\beta$-glycerophosphate. The medium was refreshed every 3 days. Recombinant human sFRP-3 (R \& D Systems, Minneapolis, MN, USA) was used at a concentration of $10 \mathrm{ng} / \mathrm{mL}$ in the osteogenic induction medium.

\section{2. $q R T-P C R$}

Reverse transcription was performed using TaqMan EZ RT-PCR Core Reagents (Applied Biosystems, Foster City, CA, USA) according to manufacturer's instructions with 100 ng of RNA. qRT-PCR was performed on an ABI PRISM 7000 Sequence Detector System (Applied Biosystems). Specific primers and probes are listed in Table 1. GAPDH primer and probe (TaqMan GAPDH detection reagents) were purchased from Perkin-Elmer (Waltham, MA, USA) and Applied Biosystems. Cycling conditions were $2 \mathrm{~min}$ at $50{ }^{\circ} \mathrm{C}, 30 \mathrm{~min}$ at $60^{\circ} \mathrm{C}, 5 \mathrm{~min}$ at $95^{\circ} \mathrm{C}$, and 50 cycles of $20 \mathrm{~s}$ at $95{ }^{\circ} \mathrm{C}$ and $1 \mathrm{~min}$ at $60^{\circ} \mathrm{C}$. Relative mRNA expression levels were normalized to that of $G A P D H$ and data from samples with sFRP-3 treatment were also normalized to those from samples without sFRP-3 treatment.

Table 1. Specific primer and probe sequences used for qRT-PCR.

\begin{tabular}{cccc}
\hline Gene & \multicolumn{1}{c}{ Sequence } & Accession No. \\
\hline ALP & $\mathrm{F}$ & AGAAAGCCAGGGGCACGAG & NM_000478 \\
& $\mathrm{R}$ & GGGAGTGCTTGTATCTCGGTTTG & \\
& Probe & CCTGGACCTCGTTGACACCTGGAAGAGC & \\
\hline ColI & $\mathrm{F}$ & GACAGTCATTGAATACAAAAC & NM_053356 \\
& $\mathrm{R}$ & ACGGAATTCTTGGTTAGTA & \\
& Probe & TAAGCCATCTCGCCTGCCAT & \\
\hline OC & $\mathrm{F}$ & GACTCTGAGTCTGACAAA & NM_013414 \\
& $\mathrm{R}$ & AGTCCATTGTTGAGGTAG & \\
& Probe & CATCCATCCATTCCACCACGC & \\
\hline Runx2 & $\mathrm{F}$ & CCTCTTATCTGAGCCAGA & NM_053470 \\
& $\mathrm{R}$ & GCAGTGTCATCATCTGAA & \\
& Probe & CATCCATCCATTCCACCACGC & \\
\hline GAPDH & $\mathrm{F}$ & GTTCCAGTATGACTCTACC & NM_017008 \\
& $\mathrm{R}$ & TCACCCCATTTGATGTTA & \\
& Probe & TTCAACGGCACAGTCAAGGC & \\
\hline
\end{tabular}

F: forward; R: reverse.

\subsection{Rat Calvarial Bone Defect Model}

Animal experiments were performed in strict accordance with protocols approved by the Animal Care Committee of Nagoya University Graduate School of Medicine (approval No. 23385 and 23414). Male Wistar/ST rats (10 weeks old; $n=8$ ) were anesthetized by intraperitoneal injection of pentobarbital (Somnopentyl; Kyoritsu Seiyaku, Tokyo, Japan) (20 mg/kg body weight). Two circular 
full-thickness bone defects $(5 \mathrm{~mm}$ in diameter) were made in the calvarial bone using a trephine bur and were irrigated with saline to remove bone debris. The experimental materials were then transplanted into the defects (Figure 4). Atelocollagen (Terudermis; Olympus Terumo Biomaterials, Tokyo, Japan), which was cut into the desired form, was resuspended with hMSCs (with or without sFRP-3 treatment; $1 \times 10^{5} /$ defect) in PBS. We defined the following groups based on the implanted materials: sFRP(-), hMSCs/Terudermis and sFRP(+), hMSCs/sFRP-3/Terudermis. Rats were sacrificed at 2, 4 or 8 weeks after transplantation ( $n=5$ per group).

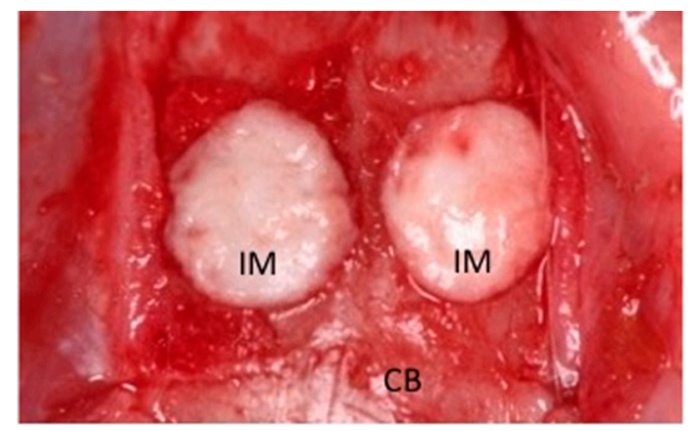

Figure 4. Experimental materials implanted into a rat calvarial bone defect. $\mathrm{CB}$, Calvarial bone; IM, Implanted material.

\subsection{Radiographic and Histological Analysis}

Surgical sites were dissected, fixed in 4\% paraformaldehyde, and subjected to micro-CT analysis using the R_mCT system (Rigaku, Tokyo, Japan). The X-ray source operated at $90 \mathrm{kV}$ and $88 \mu \mathrm{A}$, scan time $2 \mathrm{~min}$, image range $\varphi 24 \mathrm{~mm} \times 24 \mathrm{~mm}$ and voxel size $50 \mu \mathrm{m} \times 50 \mu \mathrm{m} \times 50 \mu \mathrm{m}$. The X-ray tube and flat panel rotate around the specimen to take cross-sectional X-ray CT images. The images were reconstructed on a personal computer using I-VIEW software (J. Morita, Kyoto, Japan) and analyzed using TRI/3D-BON 64 software (RATOC, Tokyo, Japan). After radiological assessment, explants were decalcified with K-CX solution (Falma, Tokyo, Japan) and dehydrated in a graded series of ethanol, cleared with xylene, and embedded in paraffin. Specimens were cut sagittally to obtain $3-\mu \mathrm{m}$-thick histological sections that were stained with hematoxylin and eosin.

\subsection{Statistical Analysis}

All experiments were conducted in triplicate and repeated at least twice. Group means and standard deviations were calculated for each measured parameter. Statistical differences were evaluated with Tukey's honestly significant difference test. $p<0.05$ was considered statistically significant.

\section{Conclusions}

sFRP-3 was shown to regulate osteogenic differentiation of hMSCs and promote bone regeneration in a rat calvarial bone defect. These findings suggest that sFRP-3 can be novel therapeutic agent for bone regeneration. 


\section{Supplementary Material}

Supplementary materials can be found at http://www.mdpi.com/1422-0067/16/10/23250/s1.

\section{Acknowledgments}

We would like to thank Editage (www.editage.jp) for English language editing. This work was supported in part by a Grant-in-Aid for Young Scientists (B) (No.1979154) from the Ministry of Education, Culture, Sports, Science, and Technology of Japan.

\section{Author Contributions}

Wataru Katagiri designed the experiment; Wataru Katagiri, Masashi Osugi, and Takamasa Kawai conducted the experiments and statistical analyses; Wataru Katagiri wrote the manuscript and Hideharu Hibi revised the manuscript.

\section{Conflicts of Interest}

The authors declare no conflicts of interest.

\section{References}

1. Shimazu, C.; Hara, T.; Kinuta, Y.; Moriya, K.; Maruo, Y.; Hanada, S.; Minagi, S. Enhanced vertical alveolar bone augmentation by recombinant human bone morphogenic protein-2 with carrier in rats. J. Oral Rehabil. 2006, 33, 609-618.

2. Herford, A.S.; Boyne, P.J. Reconstruction of mandibular continuity defects with bone morphogenic protein-2 (rhBMP-2). J. Oral Maxillofac. Surg. 2008, 66, 616-624.

3. Kawasaki, K.; Aihara, M.; Honmo, J.; Sakurai, S.; Fujimaki, Y.; Sakamoto, K.; Fujimaki, E.; Wozney, J.M.; Yamaguchi, A. Effects of reconmbinant human bone morphogenetic protein-2 on differentiation of cells isolated from human bone, muscle, and skin. Bone 1998, 23, 223-231.

4. Perri, B.; Cooper, M.; Lauryssen, C.; Anand, N. Adverse swelling associated with use of rh-BMP-2 in anterior cervical discectomy and fusion: A case study. Spine J. 2007, 7, 235-239.

5. Shields, L.B.; Raque, G.H.; Glassman, S.D.; Campbell, M.; Vitaz, T.; Harpring, J.; Shields, C.B. Adeverse effects associated with high-dose recombinant human bone morphogenetic protein-2 use in anterior cervical spine fusion. Spine 2006, 31, 542-547.

6. Langer, R.; Vacanti, J.P. Tissue Engineering. Science 1993, 260, 920-926.

7. Yamada, Y.; Ueda, M.; Naiki, T.; Takahashi, M.; Hata, K.; Nagasaka, T. Autogenous injectable bone for regeneration with mesenchymal stem cells (MSCs) and platelet-rich plasma (PRP): Tissue-engineered bone regeneration. Tissue Eng. 2004, 10, 955-964.

8. Yamada, Y.; Nakamura, S.; Ito, K.; Umemura, E.; Hara, K.; Nagasaka, T.; Abe, A.; Baba, S.; Furuichi, Y.; Izumi, Y.; et al. Injectable bone tissue engineering using expanded mesenchymal stem cells. Stem Cells 2013, 31, 572-580.

9. Dale, T.C. Signal transduction by Wnt family of ligands. Biochem. J. 1998, 329 Pt 2, 209-223.

10. Akiyama, T. Wnt/ $\beta$-catenin signaling. Cytokine Growth Factor Rev. 2000, 11, 273-282. 
11. Reya, T.; Duncan, A.W.; Ailles, L.; Domen, J.; Scherer, D.C.; Willert, K.; Hintz, L.; Nusse, R.; Weissman, I.L. A role for Wnt signaling in self-renewal of haematopoietic stem cells. Nature 2003, 423, 409-414.

12. Boyden, L.M.; Mao, J.; Belsky, J.; Mitzner, L.; Farhi, A.; Mitnick, M.A.; Wu, D.; Insigna, K.; Lifton, R.P. High bone density due to a mutation in LDL-receptor-related protein 5. N. Engl. J. Med. 2002, 346, 1513-1521.

13. Little, L.D.; Carulli, J.P.; del Mastro, R.G.; Dupuis, J.; Osborne, M.; Folz, C.; Manning, S.P.; Swain, P.M.; Zhao, S.C.; Eustace, B.; et al. A mutation in the LDL receptor-related protein 5 gene results in the autosomal dominant high-bone-mass trait. Am. J. Hum. Genet. 2002, 70, 11-19.

14. Boland, M.; Perkins, G.; Hall, D.J.; Tuan, R.S. Wnt 3a promotes proliferation and surpresses osteogenic differentiation of adult human mesenchymal stem cells. J. Cell. Biochem. 2004, 93, 1210-1230.

15. De Boer, J.; Wang, H.J.; van Blitterswijk, C. Effects of Wnt signaling on proliferation and differentiation of human mesenchymal stem cells. Tissue Eng. 2004, 10, 393-401.

16. Etheridge, S.L.; Spencer, G.J.; Heath, D.J.; Genever, P.G. Expression profiling and functional analysis of Wnt signaling mechanisms in mesenchymal stem cells. Stem Cells 2004, 22, 849-860.

17. Baksh, D.; Boland, G.M.; Tuan, R.S. Cross-talk between Wnt signaling pathways in human mesenchymal stem cells leads to functional antagonism during osteogenic differentiation. J. Cell. Biochem. 2007, 101, 1109-1124.

18. Westendorf, J.J.; Kahler, R.A.; Schroeder, T.M. Wnt signaling in osteoblasts and bone diseases. Gene 2004, 341, 19-39.

19. Bodine, P.V.; Zhao, W.; Kharode, Y.P.; Bex, F.J.; Lambert, A.J.; Goad, M.B.; Gaur, T.; Stein, G.S.; Lian, J.B.; Komm, B.S. The Wnt antagonist secreted frizzled-related protein-1 is a negative regulator of trabecular bone formation in adult mice. Mol. Endocrinol. 2004, 18, 1222-1237.

20. Oshima, T.; Abe, M.; Asano, J.; Hara, T.; Kitazoe, K.; Sekimoto, E.; Tanaka, Y.; Shibata, H.; Hashimoto, T.; Ozaki, S.; et al. Myeloma cells suppress bone formation by secreting a soluble Wnt inhibitor, sFRP-2. Blood 2005, 106, 3160-3165.

21. Yamada, A.; Iwata, T.; Yamato, M.; Okano, T.; Izumi, Y. Diverse functions of secreted frizzled-related proteins in the osteoblastogenesis of human multipotent mesenchymal stromal cells. Biomaterials 2013, 34, 3270-3278.

22. Bain, G.; Müller, T.; Wang, X.; Papkoff, J. Activated beta-catenin induces osteoblast differentiation of $\mathrm{C} 3 \mathrm{H} 10 \mathrm{~T} 1 / 2$ cells and participates in BMP-2 mediated signal transduction. Biochem. Biophys. Res. Commun. 2003, 301, 84-91.

23. Gaur, T.; Lengner, C.J.; Hovhannisyan, H.; Bhat, R.A.; Bodine, P.V.; Komm, B.S.; Javed, A.; van Wijnen, A.J.; Stein, J.L.; Stein, G.S.; et al. Canonical Wnt signaling promotes osteogenesis by directly stimulating Runx2 gene expression. J. Biol. Chem. 2005, 280, 33132-33140.

(C) 2015 by the authors; licensee MDPI, Basel, Switzerland. This article is an open access article distributed under the terms and conditions of the Creative Commons Attribution license (http://creativecommons.org/licenses/by/4.0/). 